\title{
How binocular vision is shaped by early visual experience
}

We look at the world with two eyes but we see only one picture. This is possible because the brain can bring together visual signals that originate from the left and image. How the brain single how to do that is not well understood. Through a series of experiments, Drs David Fitzpatrick, Jeremy Chang and David Whitney, from Max Planck Florida Institute for Neuroscience, showed that early visual experience drives cortical networks that is critical for binocular vision.

he world that surrounds us is
made of an infinity of shapes and colours that we perceive through our eyes. The light that reflects environment falls onto the retina thin layer of tissue that lines the back of our eyes. From there, the visual information is sent to the brain, and more specifically to the visual cortex where patterns of light are transformed into the vivid sensory experience that we call sight.

The visual cortex receives information from numerous neurons, each of

which responds selectively to a specific feature in the visual scene. For example, edges and their orientation In space carry an enormous amount of information about the visua responds on a to ach neuron fesponds only to a narrow range edges activate some neurons, vertical edges activate other neurons and still other neurons respond to different orientations in between. The visual cortex gathers all the incoming information and, depending on which neurons are activated, reconstitutes visual representations of the surrounding environmen

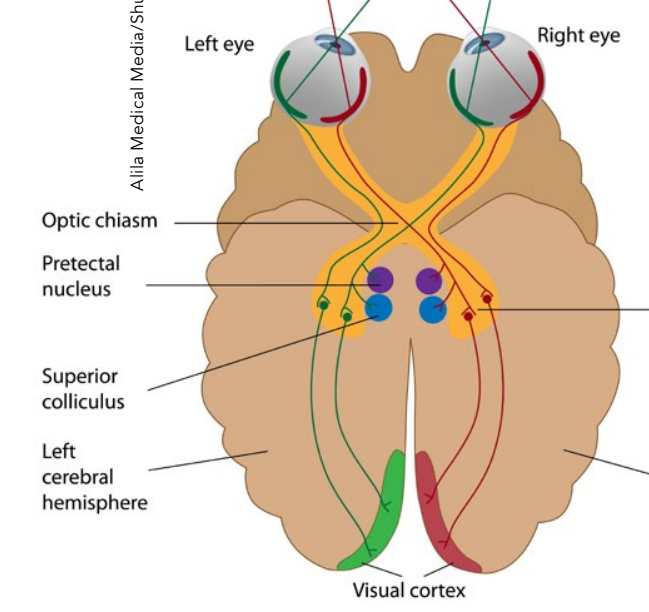

bring together the signals that come ingle unf and right eyes to create The association of the inputs from The association of the inputs from degree of precision. It is so precise that a neuron that responds to a certain orientation when the left eye is stimulated will respond selectively to the same orientation when the right eye stimulated.

What has been missing is a clear understanding of the developmenta mechanisms that are responsible for uniting the inputs from the two eyes, Davad Wh, Dr Jeremy Chang and Dr Institute for No a the Max Planck Florida by conducting ascience aimed to fill

\section{EARLY VISUAL EXPERIENCE}

\section{AND ALIGNMENT}

The first question that the team of

the role of visual experience in the

development of vision: is visual

experience required for the inputs from

the two eyes to be aligned, or does

the brain innately know how to create

The team has approached this question in the ferret. Ferrets have a well-

organised visual cortex with a modular

structure. A modular system is subdivided

into repeated units that operate

independently; in a modular visual cortex

Neurons are not randomly positioned.

nearby neurons have similar orientation
preferences - for example, neurons that single unified binocular representation?

Neurons arons have difterent functions.

Calcium imaging of neuronal activity highlights the two-step process of binocularly aligned representation development. Before visual experien
orderly but distinct networks sand mismatched cellular responses develop for each eye. Subsequently, visual experience drives network-wide

respond to horizontal edges are spatially

close to neurons that respond to almost

horizontal edges.

Dr Fitzpatrick and his collaborators use

in the absence of visual experience, the brain develops orderly network representations of edge orientations (with neurons selectively responding to specific orientations and nearby preferences). Subsequently, after eye calcium imaging to visualise neurona activity: when neurons are activated,

wanted to address is: what would patterns of activity in the visual cortex of the two eyes early in development before alignment has been achieved? They used the same techniques as described above but, instead of

We look at the world with two eyes but $\begin{gathered}\text { eyes separately } \\ \text { (monocular }\end{gathered}$

be detected by we see only one picture; what both our stimulation), they combining the use of wre stimulated them fluorescent calcium eyes perceive is unified into a single simultaneously

sensors and imaging techniques. So-called

representation.

then be visualised, giving information bout the localisation of active neurons.

More specifically, the researchers

ded calcium imaging to visualise the different patterns of activity that are prior to thed with different orientations by visually stimulating the two eyes separately, they observed that, like in the mature visual cortex, different neurons responded to different orientations, and nearby neurons had similar orientation preferences. However, patterns of activity produced by stimulation of the left eye with a single orientation were different from the patterns produced by the same stimulus orientation presented to the right eye. This suggests that develop in a two-step process. First opening these network representations reorganise to give rise to aligned in mature animals.

With additional experiments, Dr Fitzpatrick and his team confirmed that alignment of the inputs from the two eyes requires visual experience. The first week after eye opening cortical dev a critical period for period of time, visual experience drives the alignment of monocular network representations. They conclude that early visual experience is critical for proper development of vis networks that support binocu

A THIRD REPRESENTATION stimulation). Whil they had showed that monocular stimulation led to two diferent representations for the that binocular stimulation led to the appearance of a third distinct representation.

By tracking these three representations across time, they discovered that the early binocular epresentation was more stable than presentations, appearing most similar to the mature, unified visual experience. The researchers binocular representation and by binocular representation and, by doing so, guides the reorganisation pracess that resuls in the alignment coherent network. suggest that early binocular visual 


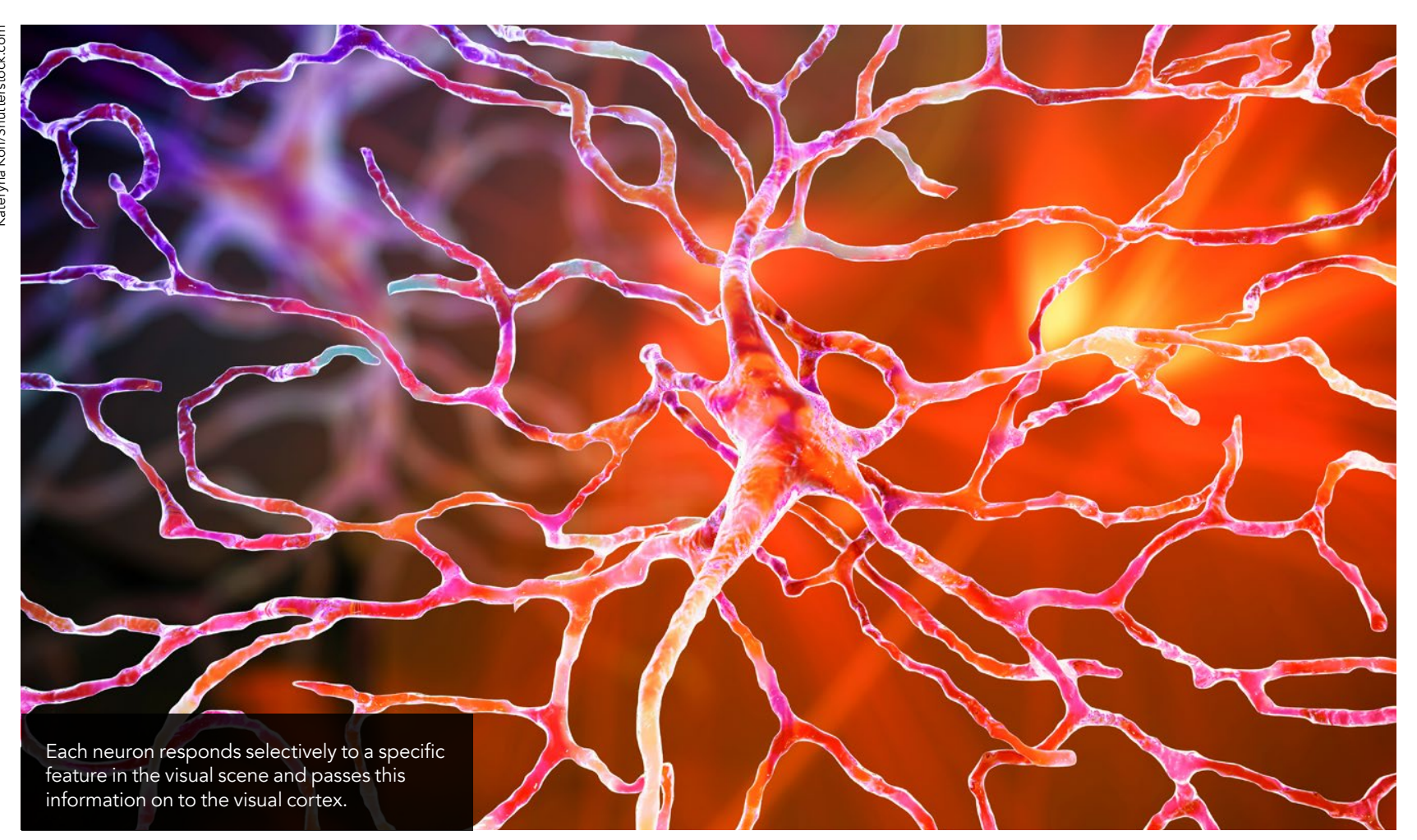

CHANGES IN NEURONS' PREFERRED ORIENTATION From the knowledge that early visual experience guides the cortical
reorganisation process arises a third reorganisation process arises a third scale? The researchers hypothesised that changes in network structure happening during the reorganisation process must reflect changes in the response properties of single neurons.

To answer this question, they used two-photon imaging. Combined with thorescent calcium sensors, this imaging activity of individul neurons. Consistent with the previous observations made at the network scale, individual neurons" orientation preferences prior to the onset of visual experience were different for the left and the right eyes. This was rectified during the reorganisation process as visual experience induced changes in preferred orientation: once network representations are aligned an individual neuron that responds to a specific orientation when the left eye is stimulated will respond to the same orientation when right eye is stimulated.

In their next studies, Dr Fitzpatrick and his team are now planning to

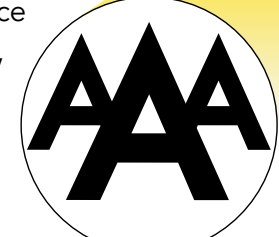

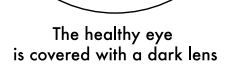

This knowledge is critical for addressing visual disorders that arise from early abnormalities in visual experience.

the synaptic scale. Their aim will be to experience-dependent alignment of identry precisely the components of the cortical networks.

the mechanisms that enable this change. This knowledge is critical for IMPACT OF THE RESEARCH addressing visual disorders that arise The res of early abnormalities in visual Plenck Florida Instide for Ne Max will provide a greater understanding happens when one eye eye " of the mechanisms responsible for the shares visual experience with the other and is thus unable to build a strong link to the brain, which results in poor vision for the affected eye and more reliance on the "good" eye.

Besides, experience-guided alignment of cortical networks is likely critical not only for vision but for a broad range of other brain functions - sensory, motor and cognitive - that are optimised for an effective navigation and interaction with our world. Identifying those aspects of brain circuitry that depend on early experience for proper alignment and understanding the could offer insights into a host of eurodevelopmental disorders whose Amblyopia means that one eye is no longer neurodevelopmental disorders
sharing visual experience with the other.
causes are still largely unknown.

\section{Behind the Research}

Dr David

Fitzpatrick

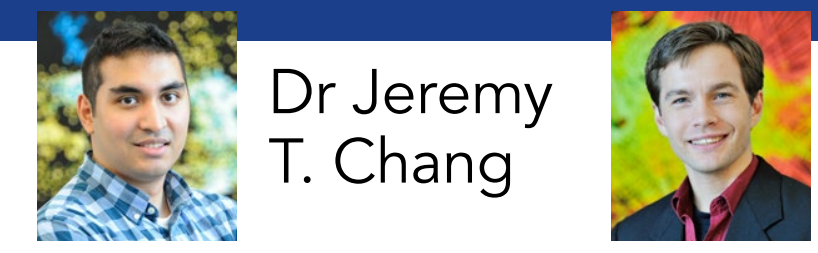

Dr David

Whitney

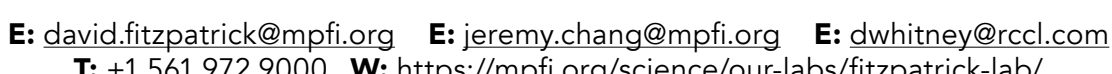

\section{Research Objectives}

Dr Fitzpatrick, Dr Chang and Dr Whitney investigate the developmental mechanisms responsible for binocular vision, offering new insights into the causes of a variety of neurodevelopmental disorders.

\section{Detail}

David Fitzpatrick

Max Planck Florida Institute for

Neuroscience

Functional Architecture and

Developme
Cortex Lab

1 Max Planck Way

USA

Bio

David Fitzpatrick is CEO and

Scientific Director at the Max Planck

Florida Institute for Neuroscience.

He is internationally recognised as

a leader in systems neuroscience,

\section{References}

Chang, J.T., Whitney, D., and Fitzpatrick, D. (2020). Experience-Dependent Reorganization Drives

Development of a Binocularly Unified Cortical

Representation of Orientation. Neuron, 107(2), neuron.2020.04.022

Fitzpatrick, D. (2020). Early visual experience drives precise alignment of cortical networks critical for binocular vision. [online] Max Planck Florida Institute for Neuroscience. Available at. https://mpti.org/early-visual-experiencedrives-precise-alignment-of-cortical-networks-critical-forbinocular-vision/ [Accessed 13 August 2020]

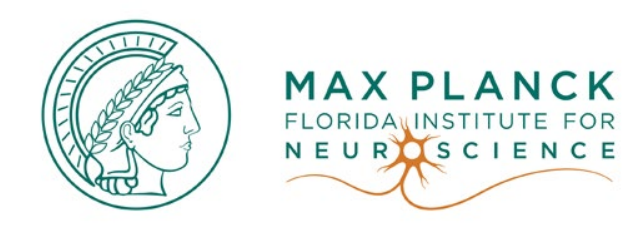

David Whitney joined the Fitzpatrick ab as a post-doc in 2013. Combining cutting-edge optical methods research focused on understanding how neural response properties emerge with the developing brain networks of visual cortex. In 2019, he Data Scientist for Data Analytics \& Al at the Royal Caribbean Group.

Funding

- Max Planck Florida Institute for

Neuroscience

\section{Personal Response}

What inspired you to conduct this research? II Networks of neurons in cerebral cortex give rise to the proper function of sensory processing, moto do not have a clearunderstanding of the that we experience-dependent versus experience-ind that processes play in the development of these networks. Visual cortex is an ideal system to investigate the role of experience-dependent processes, because, at the time of eye opening, the animals sensory experience goes have highlighted the remarkable degree of or findings early in development, and the importance of experience
in the reorganisation of these networks. 